\section{Re: Myocardial Infarction Associated with Adderall XR and Alcohol Use in a Young Man}

To the Editor: I appreciated the information in this article, ${ }^{1}$ as one who specializes in the diagnosis and treatment of attention deficit/hyperactivity disorder. I am curious whether the drug screen urinalysis performed in this case distinguished between amphetamines and methamphetamine; the article noted the patient's sample was positive for methamphetamine.

One patient of mine experienced legal problems when a routine urinalysis in the workplace resulted in positives for both amphetamine and methamphetamine; she was taking plain dextroamphetamine for her attention deficit disorder. The head of the testing laboratory assured me that their test distinguished between these agents and that a positive methamphetamine result suggested abuse whereas the positive result for amphetamines in her case was expected.

I believe the distinction is important to make in order to identify what may be a major complicating condition in a patient, namely substance abuse involving methamphetamine. In the case cited, substance abuse is evident for alcohol, but not for methamphetamines necessarily (though the Adderall was being misused).

I am unaware whether amphetamines metabolize to methamphetamine in the body, resulting in a "false positive" for methamphetamine solely from the use of amphetamines in an appropriate manner. With some drug monitoring systems making the distinction between the two, it seems unlikely.

I welcome any comments on the above.

David Pomeroy, MD, FAAFP

Brain Health NW

Bellevue, Washington

drdave@brainhealthnw.com

\section{Reference}

1. Jiao X, Velez S, Ringstad J, Eyma V, Miller D, Bleiberg M. Myocardial infarction associated with Adderall XR and alcohol use in a young man. J Am Board Fam Med 2009; 22:197-201.

doi: 10.3122/jabfm.2009.05.090048

The above letter was referred to the author of the article in question, who offers the following reply.

\section{Response: Re: Myocardial Infarction Associated with Adderall XR and Alcohol Use in a Young Man}

To the Editor: Thank you for the comment. The drug screening test cited in the article is the standard 9-panel urine screening test. It doesn't distinguish between am- phetamine and methamphetamine and reports as methamphetamine. There is, however, a more specialized test that can detect only methamphetamine. To my knowledge, methamphetamine is not one of intermediate metabolites of either amphetamine or dextroamphetamine.

Xiangyang Jiao, MD

Monarch Health

Columbus, Ohio

xyjiao111@yahoo.com

doi: 10.3122/jabfm.2009.05.090068

\section{Re: Effectiveness of Vitamin $B_{12}$ in Treating Recurrent Aphthous Stomatitis: A Randomized, Double-Blind, Placebo- Controlled Trial}

To the Editor: I read with interest the original research article, "Effectiveness of Vitamin $\mathrm{B}_{12}$ in Treating Recurrent Aphthous Stomatitis: A Randomized, Double-Blind, Placebo-Controlled Trial" by Volkov et al. ${ }^{1}$ As noted, this study suggests that daily $1000 \mathrm{mcg}$ cyanocobalamin placed under the tongue may be preventive for recurrent aphthous stomatitis (RAS) after 5 months of use. Cyanocobalamin is not bioactive and must first be converted in the body to a bioactive form before it can be of benefit.

I would like to draw your attention to another study published by myself and Jeff Haley titled "Effect of Bioactive $B_{12}$ in Adhering Discs on Aphthous Ulcers." This study, published October 2008 in Inside Dentistry with commentary by Howard E. Strassler, DMD, ${ }^{2}$ suggests that $500 \mathrm{mcg}$ bioactive methylcobalamin, when delivered daily into saliva via adherent discs that are adhered to the buccal side of a tooth and allow for a time release over 20 to 40 minutes (Avamin Melts, OraHealth, Bellevue, WA), may also be effective with respect to perceived improvement (reduced pain and duration) but with this occurring in as little as 4 weeks. This pilot study included a double-blinded, placebo-controlled trial $(n=15)$ and a nonblinded A-B-A assessment $(n=16)$.

It seems from these 2 studies that daily use of methylcobalamin time-released into saliva to allow for mucosal absorption may be an effective strategy for reducing the number, duration, and pain of recurrent aphthous ulcers, regardless of the level of underlying serum vita$\min \mathrm{B}_{12}$ levels.

Jeff Burgess, DDS, MSD

Oral Care Research Associates LLC Seattle, Washington jeffreyaburgess@hotmail.com

\section{References}

1. Volkov I, Rudoy I, Freud T, et al. Effectiveness of vitamin $B_{12}$ in treating recurrent aphthous stomatitis: a randomized, 
double-blind, placebo-controlled trial. J Am Board Fam Med 2009;22:9-16.

2. Burgess JA, Haley JT. Effect of bioactive B12 in adhering discs on aphthous ulcers. Inside Dentistry October 2008; $60-4$.

doi: $10.3122 /$ jabfm.2009.05.090083

The above letter was referred to the author of the article in question, who offers the following reply.

\section{Response: Re: Effectiveness of Vitamin $B_{12}$ in Treating Recurrent Aphthous Stomatitis: A Randomized, Double-Blind, Placebo- Controlled Trial}

To the Editor: We are satisfied to see that not only our last study, but recent research (including your own), ${ }^{1,2}$ support the conclusions of our previous observations ${ }^{3,4}$ as well. I have some commentaries on your remarks.

In my opinion, your adhering discs as well as sublingual tablets, injections, swallowed tablets, and intranasal sprays could be effective for many conditions (including recurrent aphthous stomatitis [RAS]) in regard to the effect of vitamin (Vit) $B_{12}$ itself. Undoubtedly, the time of response depends on the chosen form of cobalamin (cyanocobalamin, methylcobalamin, or hydroxycobalamin), the mode of use, and individual dosage. For example, according to my own 6 years experience you can receive an adequate response to injections of $V$ it $B_{12}$ (cyanocobalmin!) in first 2 or $3 \mathrm{wk}$. Nevertheless, some patients dislike injections and prefer tablets. The largest drawback our study was the issue of participant compliance. RAS is not a life-threatening disease, and therefore some patients even refused sublingual tablets.

\section{The Mechanism of Successful Treatment Is Still Unclear}

I disagree with the claim that the positive effect of $\mathrm{Vit}$ $\mathrm{B}_{12}$ on RAS is related to its local action on the buccal mucosa. How can you explain the response to parenteral treatment? I presume there is a generalized effect of Vit $\mathrm{B}_{12}$, and we hold a "working hypothesis" which, in our opinion, could explain this phenomenon. Vit $\mathrm{B}_{12}$ has unique yet obscure and unrecognized function. We assume that there are universal, interchangeable (as required) biologically active substances that regulate different systems of our body and provide homeostasis. We propose that one of these substances is Vit $B_{12}$. Perhaps Vit $\mathrm{B}_{12}$ can correct defects caused by other biological substances. We call this phenomenon the "Master Key effect." In summary, this fascinating and unelucidated topic definitely demands further research to disclose the underlying secrets nature set in this field.

Ilia Volkov, MD

Ben-Gurion University of the Negev Beer-Sheva

Beer-Sheva, Israel r0019@zahav.net.il

\section{References}

1. Burgess JA, Haley JT. Effect of bioactive B12 in adhering discs on aphthous ulcers. Inside Dentistry 2008;4:60-4.

2. Gulcan E, Toker S, Hatipoğlu H, Gulcan A, Toker. A Cyanocobalamin may be beneficial in the treatment of recurrent aphthous ulcers even when vitamin B12 levels are normal. Am J Med Sc 2008;336:379-82.

3. Volkov I, Rudoy I, Abu-Rabia U, Masalha T, Masalha R. Recurrent apthous stomatitis responsive to vitamin B12 treatment. Can Fam Phys 2005;51:844-5.

4. Volkov I, Rudoy I, Peleg R, Press Y. Successful treatment of recurrent aphthous stomatitis of any origin with vitamin B12 (irrespective of its blood level). Internet J Fam Pract 2007;5.

5. Volkov I, Yan P, Inna R. Vitamin B12 could be a "master key" in the regulation of multiple pathological processes. J Nippon Med Sch 2006;73:65-9.

doi: 10.3122/jabfm.2009.05.090161

\section{Re: Postepidural Headache: How Late Can It Occur?}

To the Editor: I applaud the author's enthusiasm to contribute to the medical literature; however, a number of issues detract from the validity of the published data and any conclusions that may be drawn from it. ${ }^{1}$ Although Dr. Reamy suggests that this is the first case to demonstrate the onset of postdural puncture headache (PDPH) beyond the well-accepted normal range of 1 to 7 days after epidural puncture, a range of 1 to 12 days has previously been reported in at least one case series as well as in editorials and review articles. ${ }^{2-4}$

According to the cited meta-analysis, the incidence of $\mathrm{PDPH}$ in an obstetric population is roughly $0.75 \%$ and occurs when there is an accidental entry into the intrathecal space while attempting epidural placement. ${ }^{5}$ In this case, epidural placement was uneventful-ie, without dural puncture-and thus a mechanism for entry into the intrathecal space is unclear. Furthermore, it is impossible to place an epidural catheter through a 25 -gauge needle; this is the instrument most often used to provide intrathecal analgesia. ${ }^{3}$ It is unlikely that the patient received analgesia through this route because the duration of action ( $>4$ hours) is beyond the abilities of intrathecal medications at conventional doses. Epidural catheters are typically placed through 16- to 18-gauge Tuohey needles.

Another major issue that is not adequately addressed in this report is the fact that the patient underwent a diagnostic lumbar puncture in the emergency department. Most emergency department lumbar puncture kits include a 20-gauge spinal needle, which carries a $40 \%$ risk of $\mathrm{PDPH}$ in the obstetric population. ${ }^{3}$ Moreover, in the setting of an existing symptomatic dural puncture, further drainage of cerebrospinal fluid exacerbates symptoms. The patient's symptoms were apparently improved with intravenous analgesics, antiemetics, and fluid to the point that she was discharged from the hospital after the diagnostic procedure. Interestingly, the patient's symptoms worsened significantly the day after intervention in 